\title{
Qualidade do sono e sonolência excessiva diurna em acadêmicos de medicina
}

\author{
Sleep quality and daytime sleepiness in medical students
}

Calidad del sueño y somnolencia diurna en estudiantes de medicina

Maria Cláudia Soares Oliveira ${ }^{1}$, Darah Fontes da Silva Assunção* ${ }^{1 *}$, Fábio de Azevedo Gonçalves', Fideralina Augusta da Silva Paes ${ }^{1}$, Rafael de Azevedo Silva ${ }^{1}$, Lorena Fecury Tavares ${ }^{1}$, Natália Monique Costa Caribe ${ }^{1}$, Pedro Aleixo Nogueira ${ }^{2}$, Ana Carolina Damasceno Corrêa ${ }^{1}$, Karolyne Bezerra de Oliveira ${ }^{1}$.

\section{RESUMO}

Objetivo: Avaliar a qualidade de sono e o grau de sonolência excessiva diurna em acadêmicos do curso de Medicina de um centro universitário no Estado do Pará. Métodos: Trata-se de estudo transversal descritivo, conduzido com 192 acadêmicos de medicina entre o primeiro e o quarto ano da graduação, investigados através de questionários: índice de Qualidade do Sono de Pittsburgh (PSQI), Escala de Sonolência de Epworth (ESE) e sociodemográfico. A estatística analítica foi realizada através dos Testes G e Qui-Quadrado no programa BioEstat 5.5. Resultados: Houve predominância de baixa qualidade do sono, com $29,7 \%$ de portadores de distúrbios do sono. Ao longo dos anos, a proporção de sono ruim aumenta. A ESE indicou ausência de sonolência na maioria dos estudantes, com um aumento na proporção de ausência de sonolência ao longo dos anos. Ressalta-se que essa discrepância pode estar relacionada ao aumento do consumo de substâncias estimulantes avaliado pelo estudo. Não houve significância em relação às diferenças de gênero e estado civil. Conclusão: Observou-se incidência significante de estudantes com qualidade ruim do sono, incluindo portadores de distúrbios do sono. Em contraste, o estudo demonstrou sonolência excessiva diurna na minoria dos estudantes.

Palavras-chave: Transtornos do sono-vigília, Estudantes de medicina, Sonolência.

\begin{abstract}
Objective: To evaluate the sleeping quality and the excessive daytime sleepiness degree in medical students at a university center in the state of Pará. Methods: This is a descriptive cross-sectional study, conducted with 192 medical students attending the first the four years of graduation, investigated through questionnaires: Pittsburgh Sleeping Quality Index (PSQI), Epworth Sleepiness Scale (ESE) and sociodemographic. Analytical statistics were performed using the $\mathrm{G}$ and Chi-Square Tests in the BioEstat 5.5 program. Results: There was a predominance of low sleeping quality, with $29.7 \%$ of patients with sleeping disorders. Over the years, the proportion of poor sleeping has increased. The ESE indicated absence of sleepiness over most students, with an increase in the proportion of absence of sleepiness over the years. It is pointed out that this discrepancy may be related to the increased consumption of stimulating substances assessed by the study. There was no significance regarding gender and marital status differences. Conclusion: There was a significant incidence of students with poor sleeping quality, including those with sleeping disorders. In contrast, the study showed excessive daytime sleepiness in the minority of students.
\end{abstract}

Key words: Sleep wake disorders, Students medical, Sleepiness.

\footnotetext{
${ }^{1}$ Centro Universitário Metropolitano da Amazônia (UNIFAMAZ), Belém - PA.

*E-mail: darah_fsa@hotmail.com

${ }^{2}$ Centro Universitário do Pará (CESUPA), Belém - PA.
} 
RESUMEN

Objetivo: Evaluar la calidad del sueño y el grado de somnolencia diurna excesiva en estudiantes de medicina en un centro universitario en el estado de Pará. Métodos: Este es un estudio descriptivo de corte transversal, realizado con 192 estudiantes de medicina que asistieron a los primeros cuatro años de graduación, investigado a través de cuestionarios: Pittsburgh Sleeping Quality Index (PSQI), Epworth Sleepiness Scale (ESE) y sociodemográfico. Las estadísticas analíticas se realizaron utilizando las pruebas $\mathrm{G}$ y Chi-Square en el programa BioEstat 5.5. Resultados: Predominó la baja calidad del sueño, con un $29,7 \%$ de los pacientes con trastornos del sueño. Con los años, la proporción de dormir mal ha aumentado. El ESE indicó ausencia de somnolencia en la mayoría de los estudiantes, con un aumento en la proporción de ausencia de somnolencia a lo largo de los años. Se señala que esta discrepancia puede estar relacionada con el aumento del consumo de sustancias estimulantes evaluadas por el estudio. No hubo importancia con respecto a las diferencias de género y estado civil. Conclusión: Hubo una incidencia significativa de estudiantes con mala calidad del sueño, incluidos aquellos con trastornos del sueño. Por el contrario, el estudio mostró somnolencia diurna excesiva en la minoría de estudiantes.

Palabras clave: Trastornos del sueño y vigilia, Estudiantes de Medicina, Somnolencia.

\section{INTRODUÇÃO}

As perturbações do sono podem acarretar alterações significativas no funcionamento físico, ocupacional e social do indivíduo, além de comprometer substancialmente a qualidade de vida e provocar significativos prejuízos cognitivos, ou seja, dificuldade de fixar e manter a atenção, perda de memória, diminuição da capacidade de planejamento estratégico, prejuízo motor leve, dificuldade de controlar impulsos e raciocínio prejudicado (SOUZA JC, et al., 2007; BITTENCOURT LRA, et al., 2005; MULLER MR E GUIMARÃES SS, 2007).

Nos últimos anos, foi observado uma crescente preocupação relacionada ao padrão dono em alunos graduandos. Tal demanda parte das queixas comumente apresentadas pelo grupo em questão, que são os distúrbios do sono, em especial a Sonolência Excessiva Diurna (BARDINI R, et al., 2017; MORAES JB, et al., 2019).

A privação de sono, intencional ou involuntária, promove modificações patológicas que se exteriorizam e se evidenciam frequentemente, como a sonolência diurna excessiva. Essas alterações, além de causarem aumento no risco de acidentes de trabalho e automobilísticos, também resultam em prejuízos no desempenho dos estudos, no trabalho, nas relações familiares e sociais (KABRITA C, et al., 2014; LOUREIRO CC, et al., 2011).

Nos dias atuais, os universitários estão expostos a grande estresse psicológico devido a carga horaria extensa, demandas sociais e competitividade do mercado de trabalho. Dessa forma, podem não considerar o sono como prioridade, reduzindo o tempo de sono em prol de horas extras dedicadas aos estudos e desenvolvendo hábitos de sono insatisfatórios, principalmente nas semanas em que são submetidos a testes avaliativos. Estudos com universitários em todo o mundo indicam que aproximadamente, mais da metade deles são maus dormidores (LUND H, et al., 2010; AHRBERG K, et al., 2012; ARORA R, et al., 2015).

Ainda nesse contexto, destaca-se que a quantidade de horas dormidas não representa fator determinante nesse processo. A importância da qualidade do sono torna-se instrumento fundamental por ser capaz de prover as necessidades individuais do corpo humano. (MORAES JB, et al., 2019).

Em estudo realizado em 2005, observou-se associação entre pobre qualidade do sono e aumento do risco de síndrome de Burnout, caracterizada por esgotamento de energia, falta de realização profissional e exaustão emocional, associados a sintomas depressivos e irritabilidade, principalmente em profissionais da área da saúde. 
Ademais, alterações no sono estão relacionadas ao uso de substâncias psicoativas, ansiedade e depressão. Além disso, em pacientes com depressão prévia, as queixas referentes à má qualidade de sono são maiores (MILLMAN RP, 2005, SILVA JF, et al., 2020; VASCONCELOS NA, et al., 2020; ASSUNÇÃO D, et al., 2019).

O curso de Medicina é circundado por um cenário de competitividade inerente ao processo da graduação. Dessa forma, em diversas ocasiões os graduandos sentem-se estimulado a buscar alternativas que possibilitem excelência e êxito em seu desempenho acadêmico, como o uso de substâncias psicoativas. Os universitários designam esta atitude ao uso de drogas atuar na redução do sono e aumento da concentração (MORGAN LH, et al., 2017).

Contudo, estudos recentes refutam esta ideia a partir da demonstração de que êxito e excelência no âmbito do desempenho acadêmico possuem estreita relação com melhor qualidade no sono (MIRGHANI HO, et al., 2015).

Em outro estudo, realizado na Faculdade de Medicina e Farmácia de Rabat, no Marrocos, com 600 estudantes de medicina, observou-se que estudantes do sexo feminino têm sonolência excessiva diurna com maior frequência que o sexo masculino, de acordo com a Escala de Sonolência de Epworth (ESE). Além disso, observou-se que a pobre qualidade de sono e o estresse psicológico estão relacionados com a baixa performance acadêmica dos estudantes de medicina (EL HANGOUCHE AJ, et al., 2018).

Corroborando com os achados de outro estudo, que incluiu 491 participantes, que reitera a associação de baixos escores na ESS com baixo desempenho acadêmico. Em outros estudos, no entanto, não se observou essa relação (ABDULGHANI HM, et al., 2012; AL-ZAHRANI JM, et al., 2016).

Aponta-se, ainda, que altos níveis de estresse podem ser os maiores preditores e contribuintes para a pobre qualidade do sono, associado a outros fatores, como possuir médico como familiar e preocupação com médias escolares (ALMOJALI Al, et al., 2017).

Diante disso, o presente trabalho se propõe a avaliar a qualidade de sono e a presença de sonolência excessiva diurna em graduandos do curso de medicina, possibilitando identificar os principais fatores envolvidos na sua gênese na amostra a ser analisada.

\section{MÉTODOS}

Trata-se de um estudo transversal descritivo. A população-alvo foi composta por 192 acadêmicos de medicina, escolhidos de forma aleatória entre os alunos do primeiro ao quarto ano da graduação, todos matriculados no Centro Universitário Metropolitano da Amazônia (UNIFAMAZ). A amostra foi calculada através de cálculo amostral baseado no número total de alunos nesse período por meio de amostragem aleatória simples. A coleta de dados foi realizada através de três questionários: índice de Qualidade do Sono de Pittsburgh (PSQI), Escala de Sonolência de Epworth (ESE) e questionário sociodemográfico.

O questionário PSQI é composto por 19 questões dirigidas ao paciente que abordam os seguintes itens: qualidade subjetiva do sono, latência do sono, duração do sono, eficiência habitual do sono, alterações do sono, uso de medicamentos para dormir e disfunção diurna do sono. Além disso, conta com mais 5 questões direcionadas ao parceiro(a) ou colega de quarto, que não são contabilizadas na pontuação final, mas apenas para a prática clínica. A pontuação de cada item varia de zero a vinte e um. Escore maior que cinco indica que o avaliado apresenta disfunção importante em pelo menos dois componentes, ou disfunção moderada em no mínimo três componentes.

O escore ESE contém 8 itens que podem ser pontuados de 0 a 3 pontos de acordo com a associação de situações à sonolência. Pontuação total a partir de 9 pontos indica sonolência anormal, possivelmente patológica.

O questionário sociodemográfico abordou características demográficas e comportamentais dos entrevistados, incluindo sexo, raça, estado civil, ano de graduação, prática de exercícios físicos, consumo de ansiolíticos, antidepressivos, estimulante cerebral e álcool, que foram avaliadas quanto à incidência e possível correlação com as alterações encontradas no índice PSQI e escore ESE. 
Os dados foram apresentados utilizando variáveis sob a forma de frequências absoluta e relativa, para as variáveis qualitativas, e média \pm desvio padrão, e representados em tabelas e gráficos. Os gráficos e tabelas foram construídos com as ferramentas disponíveis nos softwares Microsoft Word, Excel e Bioestat 5.5.

Todos os testes foram executados com o auxílio do software BioEstat 5.5. Para comparar as frequências observadas entre as categorias de uma mesma variável foi utilizado o teste binomial (com ajuste do p-valor para comparações múltiplas, conforme o caso).

A independência ou associação entre duas variáveis categóricas independentes foi testada pelo teste $G$ de independência. A comparação de variáveis quantitativas entre grupos foi testada pelo Teste t para dois grupos (ou seu equivalente não-paramétrico Mann-Whitney) e pelo teste ANOVA para mais de dois grupos (ou seu equivalente não paramétrico Kruskal-Wallis). Os resultados com $p \leq 0,05$ (bilateral) foram considerados estatisticamente significativos.

Todos os objetivos e procedimentos da pesquisa foram previamente esclarecidos aos participantes, os quais assinaram um termo de consentimento livre e esclarecido (TCLE). A pesquisa foi realizada com autorização do comitê de ética em pesquisa da UNIFAMAZ através do parecer 2.932.704.

\section{RESULTADOS}

Foram incluídos no estudo 192 participantes, sendo $64,6 \%$ do sexo feminino e $35,4 \%$ do masculino. A maioria era da etnia branca $(49,5 \%)$ ou parda $(43,2 \%)$ e solteiro ( $91,7 \%) .10,9 \%$ eram do primeiro ano, $42,2 \%$ do segundo, $28,6 \%$ do terceiro e $18,2 \%$ do quarto ano de Medicina (Tabela 1).

Tabela 1 - Características demográficas dos estudantes de medicina da UNIFAMAZ, entrevistados entre novembro de 2018 e janeiro de 2019, Belém - Pará. *: Diferença estatisticamente significativa. Os p-valores indicam a comparação de cada categoria com a categoria logo abaixo, por meio do teste binomial.

\begin{tabular}{cccc}
\hline Variável & $\mathbf{n}$ & $\%$ & p-valor \\
\hline Sexo & & & \\
\hline Feminino & 124 & 64,6 & $<0,001^{*}$ \\
Masculino & 68 & 35,4 & \\
\hline Raça & & & \\
\hline Branco & 95 & 49,5 & $\approx 1,000$ \\
Pardo & 83 & 43,2 & $<0,001^{*}$ \\
Negro & 10 & 5,2 & 0,309 \\
Amarelo & 4 & 2,1 & \\
\hline Estado Civil & & & \\
\hline Solteiro & 176 & 91,7 & $<0,001^{*}$ \\
Casado & 13 & 6,8 & $0,009^{*}$ \\
Divorciado & 3 & 1,6 & \\
\hline Ano Graduação & & & \\
\hline 20 & 81 & 42,2 & 0,076 \\
3o & 55 & 28,6 & 0,103 \\
4o & 35 & 18,2 & 0,180 \\
10 & 21 & 10,9 & \\
\hline
\end{tabular}

Fonte: Oliveira MC, et al., 2020.

Em relação à prática de atividades físicas e o consumo de algumas substâncias pelos participantes, observa-se que a maioria pratica exercício físico, e não consome ansiolítico, antidepressivo ou estimulante cerebral. No entanto, são equivalentes as proporções de estudantes que consomem ou não consomem bebidas alcoólicas $(p=0,248)$ (Tabela 2). 
Tabela 2 - Características comportamentais dos estudantes de medicina da UNIFAMAZ, entrevistados entre novembro de 2018 e janeiro de 2019, Belém - Pará. *: Diferença estatisticamente significativa. Os p-valores indicam a comparação de cada categoria com a categoria logo abaixo, por meio do teste binomial.

\begin{tabular}{cccc}
\hline Variável & $\mathbf{n}$ & $\%$ & p-valor \\
\hline Pratica Exercício Físico & & & $0,004^{*}$ \\
\hline Sim & 116 & 60,4 & \\
Não & 76 & 39,6 & \\
\hline Consome Ansiolítico? & & & $<0,001^{*}$ \\
\hline Não & 173 & 90,1 & \\
\hline Sim & 19 & 9,9 & \\
\hline Não & & & $<0,001^{*}$ \\
\hline Sim & 173 & 90,1 & \\
\hline Consome Estimulante Cerebral? & 19 & 9,9 & $<0,001^{*}$ \\
\hline Não & & & \\
Sim & 180 & 93,8 & \\
\hline Consome Álcool & 12 & 6,3 & \\
\hline Não & & & \\
Sim & 104 & 54,2 & \\
\hline
\end{tabular}

Fonte: Oliveira MC, et al., 2020.

A Tabela 3 exibe a classificação dos escores PSQI (qualidade de sono bom, de 0 a 5 pontos, e ruim, acima de cinco pontos) e ESE (sem sonolência excessiva diurna, com sonolência leve, moderada ou severa). Quanto ao escore PSQI, observa-se que a grande maioria (92,2\%) dos estudantes apresenta escore acima de cinco pontos (qualidade ruim de sono) e, quando ao escore ESE, sonolência diurna leve $(47,9 \%)$ ou ausência de sonolência diurna $(41,7 \%)$ prevaleceram.

Tabela 3 - Escore geral de qualidade do sono de Pittsburg e escore de sonolência diurna de Epworth dos estudantes de medicina da UNIFAMAZ, entrevistados entre novembro de $2018 \mathrm{e}$ janeiro de 2019, Belém - Pará. *: Diferença estatisticamente significativa. Os p-valores indicam a comparação de cada categoria com a categoria logo abaixo, por meio do teste binomial.

\begin{tabular}{cccc}
\hline Variável & $\mathbf{n}$ & $\%$ & p-valor \\
\hline Escore PSQI & & & \\
\hline Ruim & 177 & 92,2 & $<0,001^{*}$ \\
Bom & 15 & 7,8 & \\
\hline Escore de Epworth & & & \\
\hline Sonolência Leve (10 a 15) & 92 & 47,9 & 1,000 \\
Ausência de sonolência (0 a 9) & 80 & 41,7 & $<0,001^{*}$ \\
Sonolência Moderada (16 a 19) & 17 & 8,9 & $0,003^{*}$ \\
Sonolência Severa (20 a 24) & 3 & 1,6 & \\
\hline
\end{tabular}

Fonte: Oliveira MC, et al., 2020. 
Quanto à distribuição dos escores PSQI entre todos os alunos pesquisados, observa-se que a maioria dos alunos pesquisados possui escores de 5 a 15 pontos na escala PSQI, e que 11 discentes possuem escores elevados, de 15 a 20 pontos (Figura 1).

Figura 1 - Distribuição de escores PSQI dos estudantes de medicina da UNIFAMAZ.

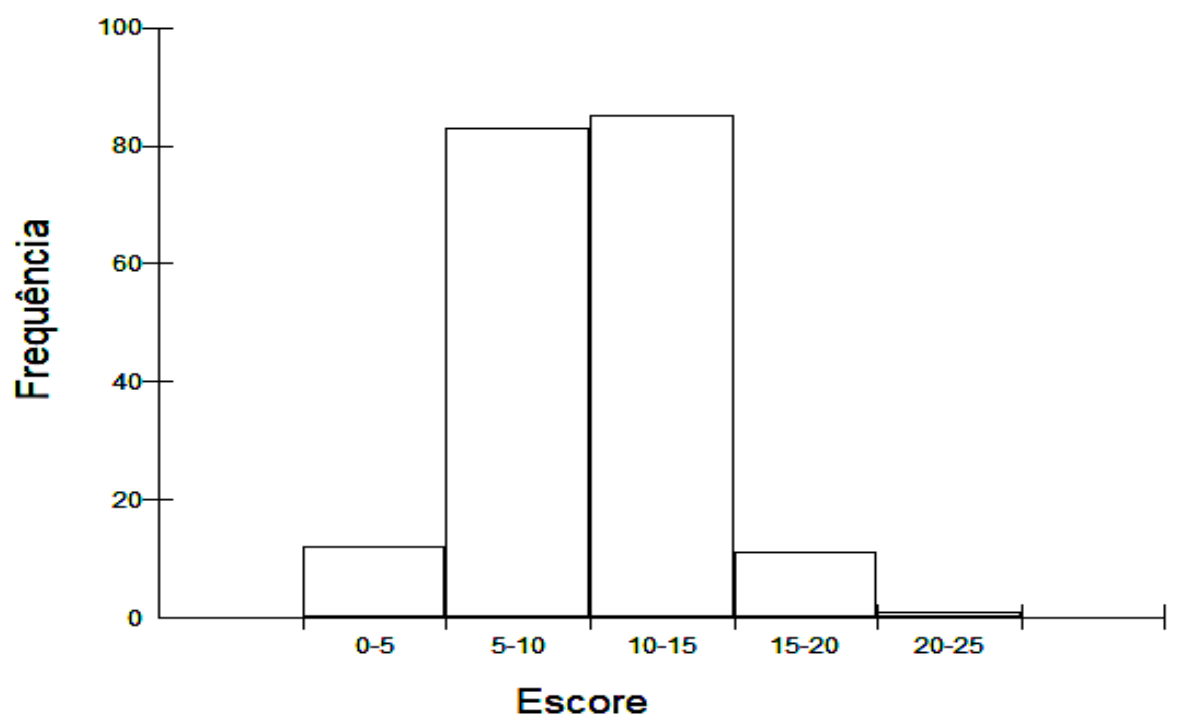

Fonte: Oliveira MC, et al., 2020.

A Figura 2 mostra a distribuição dos escores de ESE dos estudantes incluídos no estudo. Observa-se que a maioria dos estudantes possui escore ESE até o grau leve (15 pontos). A Tabela 4 exibe a comparação do escore ESE e questionário PSQI entre os quatro anos de estudo, considerando todos os estudantes. Observase que apenas o componente de disfunção diurna do questionário PSQI apresentou diferença significativa entre os grupos, sendo que no quarto ano de curso o escore foi significativamente inferior quando comparado ao primeiro, segundo e terceiro anos. Foi observado que com o passar dos anos, aumenta a proporção de alunos com ausência de sonolência excessiva diurna.

Figura 2 - Distribuição de escores de Epwort dos estudantes de medicina da UNIFAMAZ.

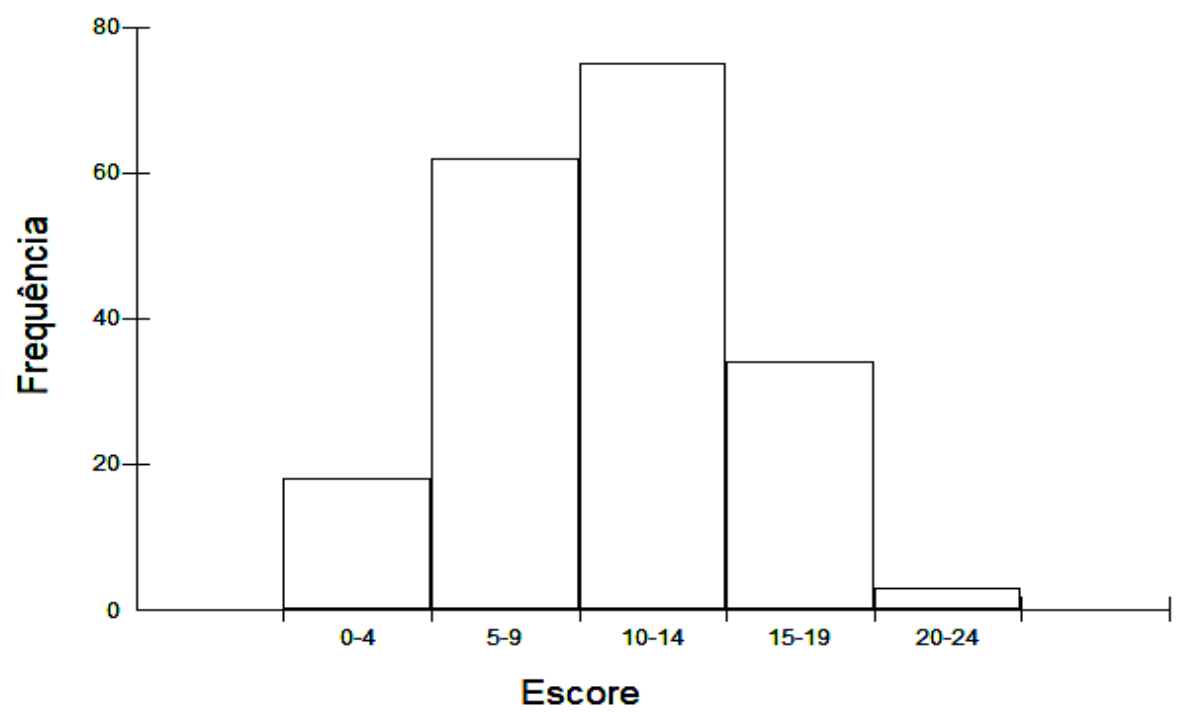

Fonte: Oliveira MC, et al., 2020. 
Tabela 4 - Índices de Qualidade do Sono de Pittsburgh e Escala de Sonolência de Epworth dos estudantes de medicina da FAMAZ, por ano de curso, entrevistados entre novembro de 2018 e janeiro de 2019, Belém Pará.

\begin{tabular}{|c|c|c|c|c|c|}
\hline Variável & $\begin{array}{l}1^{\circ} \text { Ano } \\
(n=21)\end{array}$ & $\begin{array}{l}20 \text { Ano } \\
(\mathrm{n}=81)\end{array}$ & $\begin{array}{l}\text { 30 Ano } \\
(\mathrm{n}=55)\end{array}$ & $\begin{array}{l}4^{\circ} \text { Ano } \\
(\mathrm{n}=35)\end{array}$ & p-valor \\
\hline PSQI - Geral & $10,2 \pm 3,1$ & $9,7 \pm 3,3$ & $9,9 \pm 3,3$ & $8,4 \pm 2,9$ & $0,113^{1}$ \\
\hline PSQI - Qualidade do sono & $1,7 \pm 0,7$ & $1,5 \pm 0,8$ & $1,4 \pm 0,7$ & $1,2 \pm 0,6$ & $0,088^{2}$ \\
\hline PSQI - Latência do sono & $1,4 \pm 0,9$ & $1,5 \pm 1,0$ & $1,7 \pm 0,9$ & $1,5 \pm 0,9$ & $0,340^{2}$ \\
\hline PSQI - Duração do sono & $1,8 \pm 0,9$ & $1,4 \pm 0,7$ & $1,5 \pm 0,7$ & $1,6 \pm 0,7$ & $0,201^{2}$ \\
\hline PSQI - Eficiência habitual do sono & $0,2 \pm 0,5$ & $0,3 \pm 0,7$ & $0,4 \pm 0,7$ & $0,4 \pm 0,7$ & $0,514^{2}$ \\
\hline PSQI - Alterações do sono & $1,6 \pm 0,6$ & $1,6 \pm 0,6$ & $1,6 \pm 0,6$ & $1,3 \pm 0,5$ & $0,072^{2}$ \\
\hline $\begin{array}{l}\text { PSQI - Uso de medicações para o } \\
\text { sono }\end{array}$ & $1,7 \pm 0,7$ & $1,5 \pm 0,8$ & $1,4 \pm 0,7$ & $1,2 \pm 0,6$ & $0,088^{2}$ \\
\hline PSQI - Disfunção diurna & $1,9 \pm 0,8$ & $1,9 \pm 1,0$ & $1,9 \pm 0,8$ & $1,3 \pm 0,7$ & $\begin{array}{c}0,002^{2} \\
1 \times 4,2 \times 4,3 \times 4\end{array}$ \\
\hline Escala de Epwort & $11,6 \pm 4,8$ & $10,4 \pm 5,0$ & $10,3 \pm 3,5$ & $9,7 \pm 3,4$ & $0,419^{2}$ \\
\hline
\end{tabular}

Fonte: Oliveira MC, et al., 2020.

Observa-se que não variaram significativamente os escores PSQI geral e ESE entre os sexos, mas foi significativamente maior o componente de disfunção diurna, individualmente, no sexo feminino, comparada ao sexo masculino $(p=0,028)$.

Já quando se compara os escores de qualidade do sono e de sonolência excessiva diurna entre os quatro anos de estudo, nota-se que apenas o componente de disfunção diurna do questionário PSQI apresentou diferença significativa entre os grupos, sendo que no quarto ano de curso o escore foi significativamente inferior quando comparado ao primeiro, segundo e terceiro anos. Além disso, apenas a componente latência do sono do formulário PSQI apresentou diferença significativa, sendo que os que praticam exercício possuem latência de sono superior aos que não praticam.

A comparação de escores de qualidade do sono e de sonolência excessiva diurna entre os estudantes que usam ou não bebidas diversas (café, achocolatado, energéticos ou outras substâncias com cafeína), demonstrou diferença significativa no componente duração do sono do formulário PSQI e no escore ESE, sendo que no primeiro caso, o escore de duração do sono foi estatisticamente superior entre os que apontaram não beber em relação a beber uma vez.

Em relação ao escore de ESE, a ingestão de bebidas 3 vezes aumentou o escore com relação aos casos que apontaram consumo uma vez ao dia ou nenhuma. Sendo assim, pode-se afirmar que beber esteve associado a uma diminuição do escore de duração do sono, mas a um aumento do escore de sonolência diurna. (Tabela 5). Ao realizar esta mesma análise, mas apenas para o sexo masculino ou para o feminino separadamente, não foi observada significância estatística.

Observa-se que houve diferença significativa dos escores PSQI geral e nos componentes qualidade do sono, latência do sono, uso de medicações para o sono e disfunção diurna, sendo que em todos estes casos o uso de antidepressivos foi associado a um maior escore quando comparado ao não uso. Quando comparados os escores de qualidade do sono e de sonolência excessiva diurna de acordo com o uso ou não de estimulantes cerebrais, houve diferença significativa no escore de ESE, sendo que os estudantes que usaram estimulantes cerebrais apresentaram escores significativamente superiores aos que não usaram.

Quanto à comparação dos escores de qualidade do sono e sonolência excessiva diurna, por etnia, por estado civil, e por uso ou não de ansiolíticos, em nenhum destes casos houve diferença significativa nos escores. Além disso, quando comparados os sexos masculino e feminino, observou-se que não houve variação significativa entre os escores ESSE e PSQI geral, mas foi significativamente maior o componente de disfunção diurna, individualmente, no sexo feminino, comparada ao sexo masculino. 
Tabela 5 - Índices de Qualidade do Sono de Pittsburgh e Escala de Sonolência de Epworth dos estudantes de medicina da UNIFAMAZ, por uso de bebidas, entrevistados entre novembro de 2018 e janeiro de 2019, Belém - Pará. As variáveis numéricas são exibidas como Média \pm Desvio Padrão. 1: ANOVA. 2: Teste de Kruskal-Wallis. A: 3 vezes ao dia. B: 2 vezes ao dia. C: 1 vez ao dia. D: Não consome.

\begin{tabular}{cccccc}
\hline Variável & $\mathbf{A}(\mathbf{n}=\mathbf{3 8})$ & $\mathbf{B}(\mathbf{n}=\mathbf{5 8})$ & $\mathbf{C}(\mathbf{n}=\mathbf{6 4})$ & $\mathbf{D}(\mathbf{n}=\mathbf{3 2})$ & $\mathbf{p}$-valor \\
\hline PSQI - Geral & $9,6 \pm 3,1$ & $9,7 \pm 3,5$ & $9,3 \pm 3,0$ & $10,0 \pm 3,4$ & $0,707^{1}$ \\
\hline $\begin{array}{c}\text { PSQI - Qualidade } \\
\text { do sono }\end{array}$ & $1,5 \pm 0,8$ & $1,4 \pm 0,7$ & $1,3 \pm 0,6$ & $1,6 \pm 0,8$ & $0,213^{2}$ \\
\hline $\begin{array}{c}\text { PSQI - Latência do } \\
\text { sono }\end{array}$ & $1,4 \pm 1,1$ & $1,6 \pm 0,9$ & $1,7 \pm 0,9$ & $1,4 \pm 1,0$ & $0,382^{2}$ \\
\hline $\begin{array}{c}\text { PSQI - Duração do } \\
\text { sono }\end{array}$ & $1,5 \pm 0,7$ & $1,5 \pm 0,7$ & $1,3 \pm 0,5$ & $1,9 \pm 0,8$ & $\begin{array}{c}0,011^{2} \\
\text { DxC }\end{array}$ \\
\hline $\begin{array}{c}\text { PSQI - Eficiência } \\
\text { habitual do sono }\end{array}$ & $0,2 \pm 0,5$ & $0,3 \pm 0,7$ & $0,4 \pm 0,7$ & $0,5 \pm 1,0$ & $0,559^{2}$ \\
\hline $\begin{array}{c}\text { PSQI - Alterações } \\
\text { do sono }\end{array}$ & $1,6 \pm 0,6$ & $1,7 \pm 0,6$ & $1,5 \pm 0,6$ & $1,4 \pm 0,5$ & $0,320^{2}$ \\
\hline $\begin{array}{c}\text { PSQI - Uso de } \\
\text { medicações para o } \\
\text { sono }\end{array}$ & $1,5 \pm 0,8$ & $1,4 \pm 0,7$ & $1,3 \pm 0,6$ & $1,6 \pm 0,8$ & $0,213^{2}$ \\
\hline $\begin{array}{c}\text { PSQI - Disfunção } \\
\text { diurna }\end{array}$ & $1,9 \pm 0,9$ & $1,8 \pm 0,9$ & $1,7 \pm 0,8$ & $1,7 \pm 0,8$ & $0,573^{2}$ \\
\hline $\begin{array}{c}\text { Escala de Epwort } \\
\text { PSi }\end{array}$ & $12,0 \pm 4,2$ & $10,7 \pm 3,9$ & $9,6 \pm 4,3$ & $9,3 \pm 4,8$ & $\begin{array}{c}0,018^{1} \\
\text { AxC,AxD }\end{array}$ \\
\hline
\end{tabular}

Fonte: Oliveira MC, et al., 2020.

\section{DISCUSSÃO}

O presente estudo teve como objetivo avaliar a qualidade do sono e presença de sonolência diurna. Essa temática entre estudantes universitários tem sido objeto de estudo em pesquisas recentes, associando-se alterações do sono a prejuízos acadêmicos. Para tal, diversos instrumentos vêm sendo utilizados, destacando-se dentre estes a PSQI e ESE, que possibilita a avaliação de fatores frequentes na vivência de acadêmicos, tornando-se ferramentas essenciais para a percepção da vulnerabilidade a que todos estão sujeitos, bem como a importância dessa problemática. (BENAVALENTE SB, et al., 2014; ARAÚJO MF, et al., 2014; LEMMA S, et al., 2014).

No atual estudo, onde se vê maior prevalência de participantes do sexo feminino, não foram observadas diferenças entre gêneros. No que diz respeito as taxas de sonolência excessiva diurna, foi visto diminuição destas nos anos de graduação mais avançados do curso.

Destaca-se a interferência do uso de psicoativas, notando-se escores superiores de qualidade do sono e de sonolência excessiva diurna entre os graduandos que utilizaram estimulantes cerebrais quando comparado aos que não fizeram uso.

No que diz respeito a associação qualidade do sono e de sonolência excessiva diurna entre os anos de graduação pesquisado, o presente estudo, revela que apenas o componente de disfunção diurna demonstrou diferença significativa entre os grupos, sendo que no quarto ano de curso apresentou escore significativamente inferior quando comparado aos demais anos. 
Em contraste com o presente estudo, observou-se prevalência de insônia em $28,2 \%$ de acadêmicos do curso de Medicina, com predominância no sexo feminino, no qual foi relatada maior dificuldade em manter 0 sono. Além disso, descreve-se em outro estudo, aumento progressivo da incidência de sonolência diurna excessiva em estudantes universitários de Medicina (LOAYZA HMP, et al., 2001; RODRIGUES RN, et al., 2002).

A literatura atual aponta que a sonolência diurna excessiva está associada a diversos distúrbios psiquiátricos e neurológicos, podendo causar prejuízos na qualidade de vida dos indivíduos. Destaca-se que tal advento também está relacionado ao impacto no desempenho acadêmico (LEMMA S, et al., 2014; MIRGHANI H, 2015).

As taxas de qualidade de sono ruim revelam-se elevadas em diversos estudos de acordo com a escala PSQI. Foram encontradas taxas aproximadas e maiores em outras pesquisas (SEGUNDO LVG, et al., 2017; MULLER MR e GUIMARÃES SS, 2007; RIQUE GL, et al., 2014; RIBEIRO CR, et al., 2014).

Em um determinado estudo que avaliou a qualidade subjetiva do sono, observou-se que $40 \%$ dos estudantes qualificaram-na como ruim ou muito ruim, sendo maior no $3^{\circ}$ e $4^{\circ}$ anos de graduação em relação ao $5^{\circ}$ e 6ํㅡㅁ anos. Esse fator, apesar de contraditório, talvez possa ser explicado pelo aumento do consumo de bebidas que contenham cafeína como uma forma de adaptação ao sono, que explicaria a piora da qualidade do sono com menor sonolência excessiva diurna.

É amplamente difundido que a cafeína promove estímulos que aumentam a atenção e o desempenho, com maior latência para o sono e piora na qualidade do sono. O consumo de álcool, em contrapartida, causa relaxamento e ação sedativa. Mas, seu uso excessivo pode levar à redução da qualidade do sono devido ao aumento dos despertares noturnos e estágios superficiais do sono por ativação do sistema simpático (PINHEIRO SMS, 2012; CLARK I e LANDOLT HP, 2017).

É visto na literatura contradição quanto a associação da piora da qualidade do sono e o consumo de substâncias psicoativas, destacando-se a importância de aprofundar essa investigação. Somado a isso, observa-se a necessidade de ampliação do grupo de interesse na investigação, no âmbito acadêmico, quanto a qualidade do sono visto que entre professores universitários foram encontradas associações estatisticamente significativas entre sonolência excessiva diurna e prejuízo na qualidade de vida com prevalência em 1 em cada 3 dos estudados (LOPES HS, et al., 2018; AMARO JM e DUMITH SC, 2018).

Vale destacar a importância da melhoria do sono na saúde mental em acadêmicos graduandos no curso de Medicina. A melhoria da qualidade de vida dos futuros médicos pode resultar em fortalecimento da qualidade assistencial nos diversos níveis de saúde (AZAD MC, et al., 2015).

Com isso, o advento da melhoria na qualidade do sono torna-se intimamente influenciado pela ausência de boas práticas da higiene do sono, minimizados na população estudada pelos próprios sujeitos. Nota notase escassez em ações de promoção de saúde no grupo acrescido por práticas de maus hábitos e baixa adesão de técnicas de higiene do sono.

A análise dos dados obtidos na ESE de um estudo realizado entre estudantes do curso de Medicina da Universidade Federal da Paraíba demonstrou que $81,6 \%$ dos entrevistados apresentavam sonolência excessiva diurna grave.

Foi verificada relação entre a qualidade do sono e interferências nas atividades diárias dos estudantes. Constatou-se que estudantes cuja qualidade do sono é ruim apresentam 2,42 vezes mais chance de ter uma interferência ruim ou muito ruim nas atividades diárias (SEGUNDO LVG, et al., 2017).

A qualidade do sono de graduandos em Medicina de uma Faculdade de Medicina no interior do estado de São Paulo foi analisada e descrita, observando-se comprometimentos em determinados componentes do PSQI, propondo-se a manutenção dessa investigação em diferentes regiões do país e do mundo, com a finalidade de observar o perfil desses estudantes e encorajar a transposição desses dados para as práticas de promoção de saúde (CORRÊA CC, et al., 2017). 
Os resultados concordaram com os da literatura em relação à elevada frequência de aspectos alterados da qualidade do sono, que não é interpretada como problema ou distúrbio, o que pode ocasionar repercussões nocivas à saúde. Deve incluir a interpretação dos autores sobre os resultados obtidos e sobre suas principais implicações, a comparação dos achados com a literatura, as limitações do estudo e eventuais indicações de caminhos para novas pesquisas.

\section{CONCLUSÃO}

Observou-se incidência significante de estudantes com qualidade ruim do sono, incluindo portadores de distúrbios do sono, Com piora gradual da qualidade do sono com o passar dos anos. Em contraste, o estudo demonstrou sonolência excessiva diurna na minoria dos estudantes. Possivelmente, o consumo de substâncias com cafeína levou a diminuição na duração do sono e aumento da sonolência excessiva diurna. Diversas outras variáveis foram analisadas para associação com prejuízos na qualidade do sono e a sonolência excessiva diurna em acadêmicos de medicina. Com isso, os resultados apresentados assumem relevância e tornam-se fonte de dados para realização de pesquisas futuras acerca dessa temática. Ressaltase a necessidade de pesquisas futuras com maior abrangência, visto que se considerou apenas o cenário local, com objetivo de estender a análise entre acadêmicos dos cursos de saúde, avaliando de forma mais ampla a influência de fatores internos e externos na qualidade do sono dos universitários.

\section{REFERÊNCIAS}

1. ABDULGHANI HM. Sleep disorder among medical students: relationship to their academic performance. Med Teach. 2012; 34(1): 37-41.

2. AHRBERG K, et al. The interaction between sleep quality and academic performance. J Psychiatr Res. 2012; 49: 16181622.

3. ALMOJALI Al, et al. The prevalence and association of stress with sleep quality among medical students. Journal of epidemiology and global health. 2017; 7(3):169-174

4. ARAÚJO MF, et al. Health indicators associated with poor sleep quality among university students. Rev Esc Enferm USP. 2014; 48(6):1085-92.

5. ARORA R, et al. Burnout and Sleep Quality: A Cross-Sectional Questionnaire-Based Study of Medical and Non-Medical Students in India. Cureus. 2015; 7:361.

6. ASSUNÇÃO D, et al. A ocorrência da Síndrome de Burnout em universitários de cursos da área da saúde de uma instituição privada na região Norte de Minas Gerais: um estudo transversal. Revista de Ciências Médicas e Biológicas 2019. 18(1):15-20.

7. AZAD MC, et al. Sleep disturbances among medical students: a global perspective. Journal of clinical sleep medicine. JCSM. 2015; 11:69-74.

8. BARDINI R, et al. Prevalência de sonolência excessiva diurna e fatores associados em adolescentes universitários do sul catarinenese. Arq. Catarin Med. 2017; jan-mar; 46(1): 107-124.

9. BENAVENTE SB, et al. Influence of stress factors and socio-demographic characteristics on the sleep quality of nursing students. Rev Esc Enferm USP. 2014; 48(3):514-20.

10. BITTENCOURT LRA, et al. Sonolência excessiva. RevBras Psiquiatr. 2005; 27(Supl.1):16-21.

11. BOLÉ-TOMÉ JP, et al. Prevalência de doença cardiovascular numa população de doentes com síndrome de apneia obstrutiva do sono. Rev Port Clin Geral. 2008; 24:355-361.

12. CLARK I e LANDOLT HP. Coffee, caffeine, and sleep: a systematic review of epidemiological studies and randomized controlled trials. Sleep Med Rev. 2017; 31:70-78.

13. CORRÊA CC, et al. Qualidade de Sono em Estudantes de Medicina: comparação das diferentes fases do curso. J Bras Pneumol. 2017; 43(4):285-289.

14. EL HANGOUCHE, et al. Relationship between poor quality sleep, excessive daytime sleepiness and low academic performance in medical students. Advances in medical education and practice 2018; 9, 631-638.

15. FERNÁNDEZ-MENDOZA J, et al. Nighttime sleep and daytime functioning correlates of the insomnia complaint in young adults. J Adolesc. 2009; 32(5):1059-74.

16. HYDER M. Good sleep quality is associated with better academic performance among Sudanese medical students. BMC Research Notes. 2015; 8:706-710.

17. KABRITA CS, et al. Predictors of poor sleep quality among Lebanese university students: association between evening typology, lifestyle behaviors, and sleep habits. Nat Sci Sleep. 2014; 6:11-18.

18. LEMMA S, et al. Good quality sleep is associated with better academic performance among university students in Ethiopia. Sleep Breath. 2014; 18(2):257-63.

19. LOPES HS, et al. Qualidade do Sono entre Estudantes de Enfermagem e Fatores Associados. Semina: Ciências Biológicas e da Saúde, Londrina. 2018; 39(2):129-136.

20. LOUREIRO CC, et al. Reacção paradoxal da pressão arterial ao tratamento com pressão positiva na via aérea em doentes com apneia do sono. Rev Port Pneumol. 2011; 17(2):53-8. 
21. LUND H, et al. Sleep Patterns and Predictors of Disturbed Sleep in a Large Population of College Students. J Adolesc Health. 2010; 46: 124-32.

22. MILLMAN RP. Excessive sleepiness in adolescents and young adults: causes, consequences, and treatment strategies. Pediatrics. 2005. 115(6):1774-86.

23. MIRGHANI HO, et al. Good sleep quality is associated with better academic performance among Sudanese medical students. BMC Res Notes 2015;8(706):1-5.

24. MORAES JB, et al. Sonolência excessiva diurna em alunos do último ano do curso de fisioterapia de uma faculdade privada. Revista Eletrônica Acervo Saúde. 2019; 17: e371.

25. MORGAN HL, et al. Consumo de estimulantes cerebrais por estudantes de medicina de uma universidade do Extremo Sul do Brasil. Rev Bras Educ Med 2017;41(1):102-9.

26. MULLER MR, GUIMARÃES SS. Impacto dos transtornos do sono sobre o funcionamento diário e a qualidade de vida. EstudPsicol. 2007; 24(4):519-28.

27. PINHEIRO SMS. Consumo de cafeína por doentes com síndrome de apneia obstrutiva do sono. Dissertação. Universidade de Coimbra, Coimbra, 2012;

28. RIBEIRO CR, et al. The impact of sleep quality in medical education. Rev Soc Bras Clin Med. 2014. $12(1): 8: 14$.

29. RIQUE GL, et al. Relationship between chronotype and quality of sleep in medical students at the Federal University of Paraiba, Brazil. Sleep Sci. 2014; 7(2):96-102.

30. RODRIGUES RN, et al. Daytime sleepiness and academic performance in medical students. ArqNeuropsiquiatr 2002; 60:6-11.

31. SEGUNDO LVG, et al. Aspectos Relacionados à Qualidade do Sono em Estudantes de Medicina. Revista Brasileira de Neurologia e Psiquiatria. 2017; 21(3):213-223.

32. SILVA JF, et al. Síndrome de Burnout em profissionais de Enfermagem no contexto da Atenção Básica. Revista Eletrônica Acervo Saúde, 2020; (39): e2320.

33. SOUZA JC, et al. Sonolência diurna excessiva em pré-vestibulandos. J BrasPsiquiatr. 2007; 56:184-7.

34. VASCONCELOS NA, et al. Avaliação do contexto de trabalho e burnout em residentes e preceptores. Revista Eletrônica Acervo Saúde. 2020; (12): e3387. 